\title{
O PROGRAMA DE EXTENSÃO ERUS E SUA CONTRIBUIÇÃO NA FORMAÇÃO DO ENGENHEIRO CAPIXABA
}

LorenaBacheti Bassani-lorena.bassani@aluno.ufes.br

Universidade Federal do Espírito Santo

Av. Fernando Ferrari, 514, Goiabeiras

29075-910 - Vitória - Espírito Santo

Gabriel Pietroluongo Nascimento - gabriel.nascimento.50@aluno.ufes.br

Universidade Federal do Espírito Santo

Av. Fernando Ferrari, 514, Goiabeiras

29075-910 - Vitória - Espírito Santo

Higor David Oliveira - higor.d.oliveira@aluno.ufes.br

Universidade Federal do Espírito Santo

Av. Fernando Ferrari, 514, Goiabeiras

29075-910 - Vitória - Espírito Santo

André Ferreira-andrefer@ele.ufes.br

Universidade Federal do Espírito Santo

Av. Fernando Ferrari, 514, Goiabeiras

29075-910 - Vitória - Espírito Santo

Resumo: Este documento apresenta os trabalhos da Equipe de Robótica da Ufes e como contribuíram nos últimos oito anos para a formação do engenheiro capixaba, incentivando e permitindo aos alunos da Universidade Federal do Espírito Santo desenvolverem suas habilidades em robótica, computação, mecânica e eletrônica através do método de Aprendizagem Baseada em Problemas (Problem-Based Learning).

Palavras-chave: Robótica. PBL. Engenharia.

\section{INTRODUÇÃO}

A partir do final do século XX, houve uma grande mudança nas formas como as pessoas se comunicam, trabalham, acessam informações e usam tecnologias (Duch, Groh \& Allen, 2001). O advento e a normalização do acesso à Internet apresentaram aos cidadãos brasileiros a possibilidade de acessar conhecimento de forma praticamente ilimitada, o que antes era quase exclusivo de estudiosos da área e possuía preço pouco acessível para conteúdos especializados, criando uma barreira de entrada, principalmente na área da engenharia. Contudo, essa maior acessibilidade também incorre em uma maior demanda intelectual do mercado de trabalho acerca da formação do engenheiro. Dessa forma, é necessário preparar o graduando para esse novo paradigma social. Mais do que nunca, alunos necessitam de aprender muito mais que apenas os conceitos teóricos abordados na metodologia clássica de ensino: precisam aprender como identificar as questões do mundo real e, além de propor as soluções, aprender a comunicá-las de forma efetiva. Passou-se a exigir mais do perfil do engenheiro do amanhã: 
- Pensamento crítico e capacidade de analisar e resolver problemas complexos do mundo real;

- Encontrar, avaliar e usar recursos de aprendizagem apropriados;

- Trabalhar cooperativamente em equipes e pequenos grupos;

- Demonstrar habilidades de comunicação versáteis e efetivas, tanto verbais como escritas;

- Utilizar conhecimento e habilidades intelectuais adquiridas na universidade para se tornar aprendizes contínuos.

Dessa forma, o aprendizado na engenharia se torna extremamente desafiador, e até mesmo inadequado, se for dependente apenas do modelo tradicional de ensino. Para complementar este modelo, os grupos de pesquisa e extensão se apresentam como alternativas para os futuros engenheiros, viabilizando a exposição a diversos conteúdos não contemplados no período de graduação e proporcionando aos estudantes um domínio maior das suas áreas de atuação antes mesmo da primeira experiência profissional. Dada a alta concorrência e as expectativas e demandas cada vez mais extenuantes no mercado de trabalho, as iniciativas de pesquisa e extensão proporcionam um diferencial inestimável ao engenheiro do amanhã.

É precisamente nesse contexto que o programa de extensão ERUS pode contribuir para a formação acadêmica e profissional do futuro engenheiro, à medida que proporciona contato direto com diversos aspectos necessários tanto para o desenvolvimento intelectual, expondo o membro da equipe a novos paradigmas e tecnologias, quanto para o desenvolvimento interpessoal, exigindo de todo membro habilidades de trabalho em equipe e, por vezes, apresentações a professores e estudantes externos à equipe. Essas são algumas das diversas características essenciais para formar um profissional qualificado na atualidade e, dessa forma, a ERUS atua como um catalisador de melhorias na formação do engenheiro capixaba.

\section{BREVE HISTÓRICO: DE UMA EQUIPE DE ROBÓTICAA UM PROGRAMA DE EXTENSÃO}

A Equipe de Robótica da Ufes - ERUS - foi fundada em 2012, por alunos da engenharia de computação da Universidade Federal do Espírito Santo, ex-integrantes da equipe de robótica Ufes Ultrabots, projeto do Laboratório de Robótica Educacional - LRE, fundado em 2006. Eles fundaram a ERUS em busca de desafios mais complexos, tendo como principal objetivo desenvolver e aplicar conceitos aprendidos durante os cursos de tecnologia.

Mais de 60 alunos da Ufes já passaram pela equipe durante seus 8 anos de trabalho. Estes alunos são hoje estudantes e egressos dos mais diversos cursos das áreas de engenharia e tecnologia, como: engenharia da computação, engenharia elétrica, engenharia mecânica, engenharia civil, ciência da computação e física. É possível ver algumas gerações de membros da equipe na Figura 1 e na Figura 2. 
Figura 1 - Equipe da ERUS no ano de 2013, premiada na categoria IEEE OPEN da LARC.

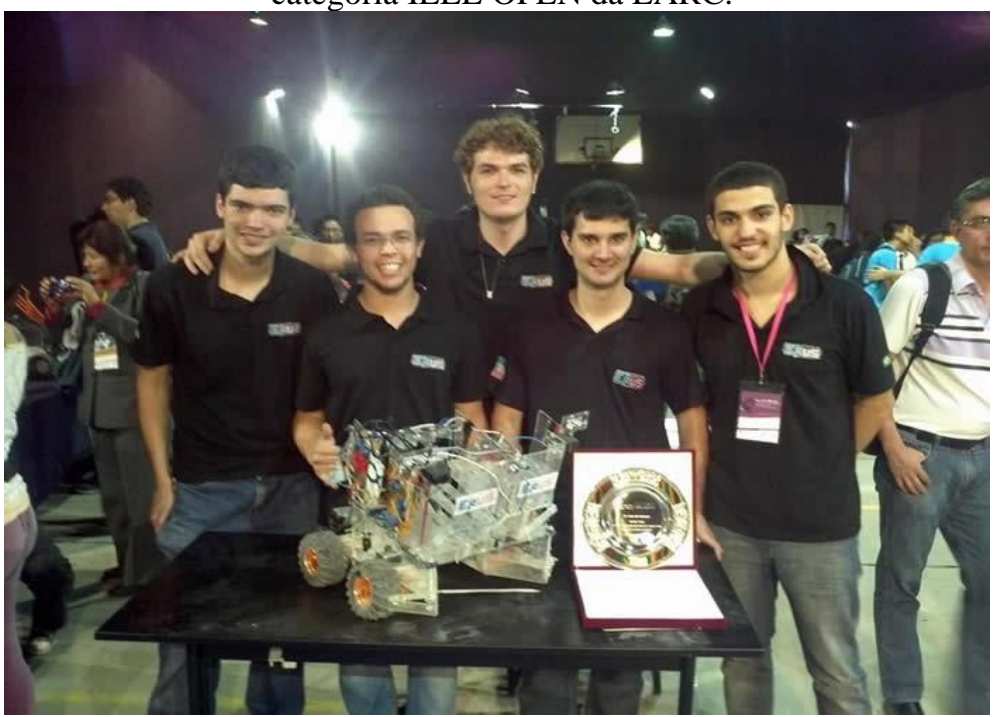

Fonte: de autoria própria.

Figura 2 - Equipe da ERUS no ano de 2019, durante o VI TRUFES.

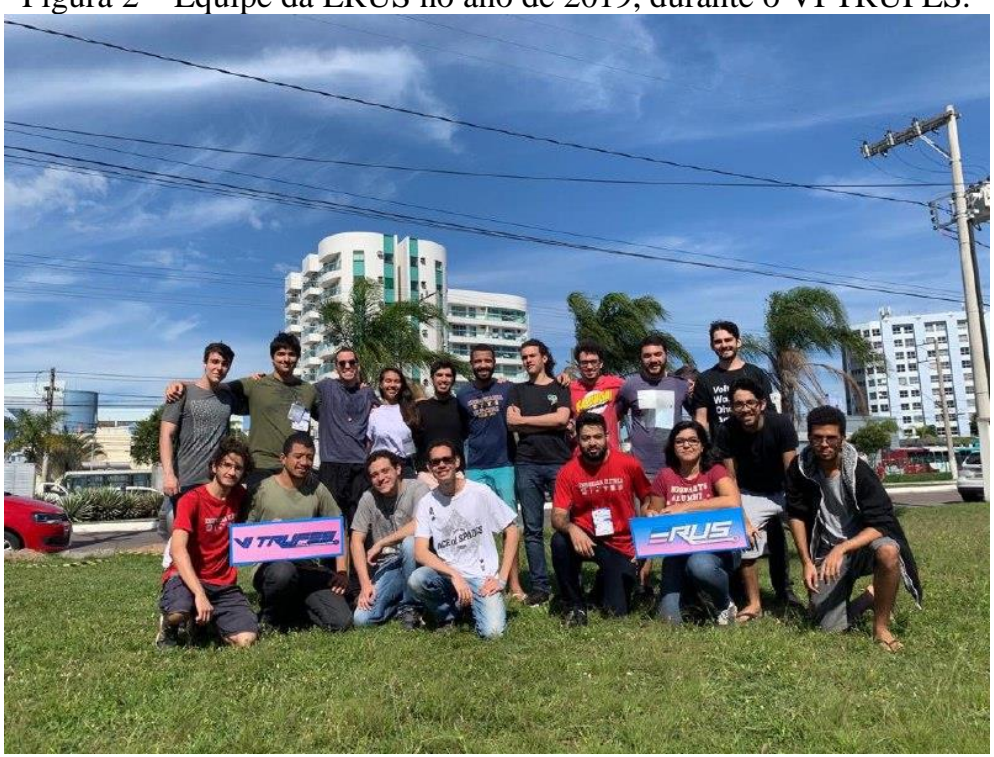

Fonte: de autoria própria.

A ERUS é um berço para a formação de futuros engenheiros e cientistas, como podemos perceber a partir do desenvolvimento e da carreira de seus egressos. Muitos participaram em grupos de pesquisa de grande relevância ou ocupam posições importantes em empresas e startups, sendo alguns de seus ex-membros amplamente conhecidos no ambiente empresarial, a exemplo de Allan Mota, fundador da loja de componentes eletrônicos Vida de Silício.

Com apoio incondicional do Programa PET Engenharia da Computação da Ufes, a ERUS inicialmente concentrou seus esforços para competir nas categorias IEEE-OPEN e IEEEVSSS na Latin American Robotics Competition (LARC), além de participar de diversas competições pela América Latina. Mais tarde, a ERUS também concentrou esforços em organização de minicursos, workshops e competições. Desde 2014, a equipe organiza o TRUFES - Torneio de Robótica da Ufes, competição de âmbito nacional voltada a incentivar alunos de ensino fundamental, médio e superior a utilizarem robótica como ferramenta para 
resolver desafios atuais. Na Figura 3 é possível ver um desses desafios: o desafio da lavanderia, utilizado no II e VI TRUFES.

Figura 3 - Categoria da Lavanderia no II TRUFES, 2015.

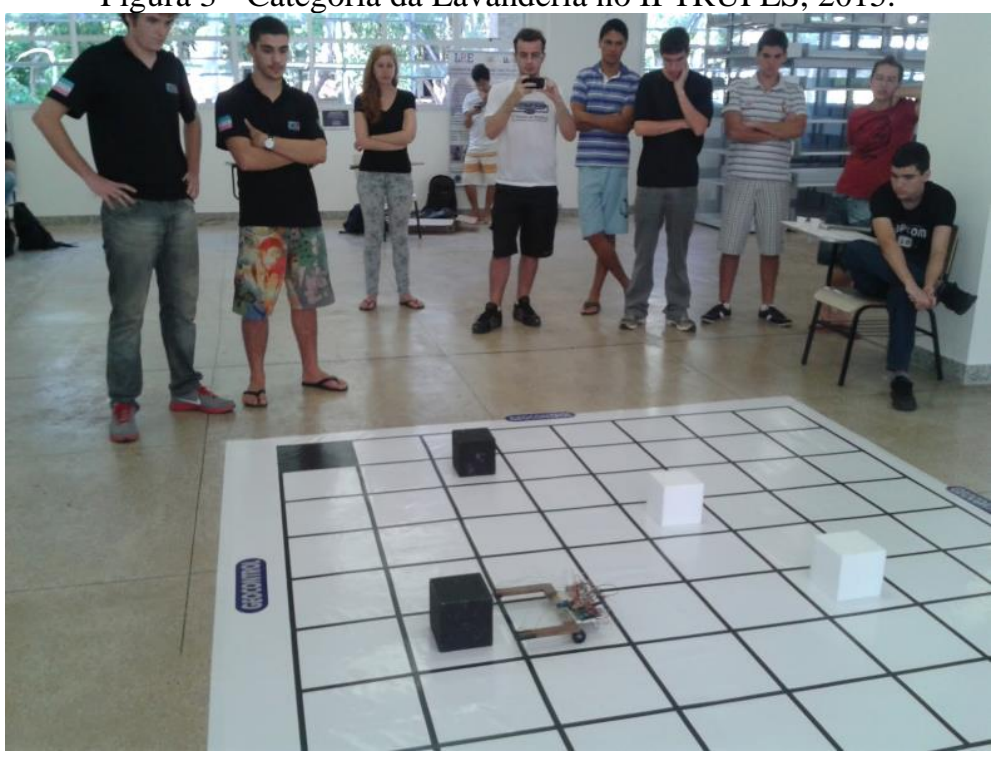

Fonte: de autoria própria.

Em 2019, a ERUS deu um novo passo em sua história ao tornar-se Programa de Extensão vinculado ao Centro Tecnológico da Universidade Federal do Espírito Santo, assumindo a importante missão de reunir diversos cursos da Ufes em prol do desenvolvimento da Robótica no estado do Espírito Santo.

\section{ERUS COMO EXTENSÃO UNIVERSITÁRIA E SUA RELEVÂNCIA PARA OS CAPIXABAS}

\subsection{Extensão: Foco na Formação do Aluno e em Ações Extramuros da} Universidade.

O conceito de extensão abrange todo o desenvolvimento do engenheiro em formação além da fronteira da universidade, seja em estágios associados à sua área de atuação ou na participação em projetos extracurriculares (RODRIGUES, 2013), como é o caso da equipe. Dessa forma, a ERUS fomenta este aspecto essencial da formação dos futuros engenheiros: a implementação de conceitos extensivos tais como os descritos anteriormente, que são imprescindíveis à formação adequada do profissional da engenharia. A equipe expande os horizontes de seus membros à medida que apresenta diversos projetos da frente extensionista, como torneios (seja como organizador ou participante) e aulas ministradas aos próprios membros ou ao público externo e apresentações envolvendo robótica de maneira lúdica para o público mais jovem e que ainda cursa o ensino médio ou até mesmo o fundamental. A ERUS possibilita o contato de jovens que, muitas vezes, não teriam condições de acessar o domínio da robótica. Desta forma, democratiza o acesso à tecnologia e ao conhecimento por meio de tais ações, assim como outros projetos de extensão da Ufes realizam, como o Introcomp, projeto que apresenta conceitos de programação a alunos de escolas públicas do Espírito Santo e organizado pelo PET Engenharia da Computação da Ufes. Recentemente, a ERUS ministrou uma aula de encerramento no projeto Introcomp envolvendo aplicações de Arduino e Processing e integrando o conteúdo apresentado regularmente pelo PET Engenharia da 
C COBENGE

"Os desafios para formar hoje o engenheiro do amanhã"

Computação no curso a novas tecnologias e possibilitando aos alunos vislumbrarem aplicações alternativas dos conhecimentos adquiridos no projeto.

\subsection{Robótica Educacional}

Anualmente, a ERUS organiza o TRUFES. Cada edição conta com um ou mais desafios propostos pela equipe no âmbito da robótica, como o "Desafio do Ogro" (no qual as equipes deveriam desenvolver um robô que conseguisse desviar de cubos coloridos em uma esteira), o "Desafio da Lavanderia" (no qual deveria ser desenvolvido um robô que, em determinada arena confeccionada pela equipe, deveria levar alguns cubos coloridos para locais prédeterminados), dentre outros.

Historicamente, o TRUFES é realizado em parceria com a SENG (Semana da Engenharia da Ufes) e com a JACEE (Jornada de Atualização em Computação, Elétrica e Eletrônica), sendo uma de suas principais atrações. O torneio atrai participantes de todas as idades, desde os mais novos, acompanhados de alguma instituição responsável, até os mais veteranos da área vindos, inclusive, de outros estados.

O TRUFES é uma das principais ações de extensão da ERUS, já que envolve praticamente todos os seus membros em sua organização e acaba por exercitar a maior parte das áreas associadas à equipe, desde o planejamento dos desafios, a confecção manual das pistas, os testes com robôs criados internamente, até o desenvolvimento de sistemas de avaliação e publicações no site associadas à divulgação do evento. $\mathrm{O}$ torneio é realizado com o empenho de toda a equipe, e é um grande vetor da área da robótica ao público geral. As Figura 4 e Figura 5 mostram a equipe, envolvidos e participantes do III e VI TRUFES, evidenciando a importância e o impacto deste evento para a robótica capixaba.

Figura 4 - Equipe, participantes e envolvidos no III TRUFES em 2016.

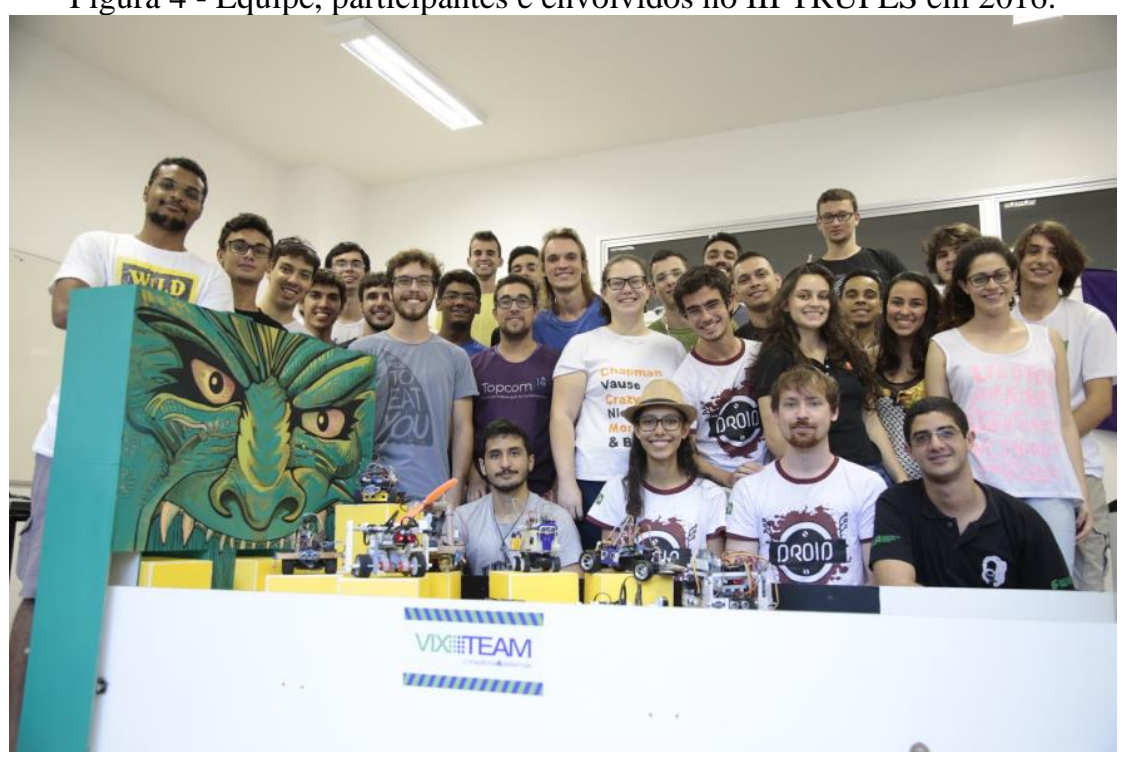

Fonte: de autoria própria. 
Figura 5 - Equipe, participantes e envolvidos no VI

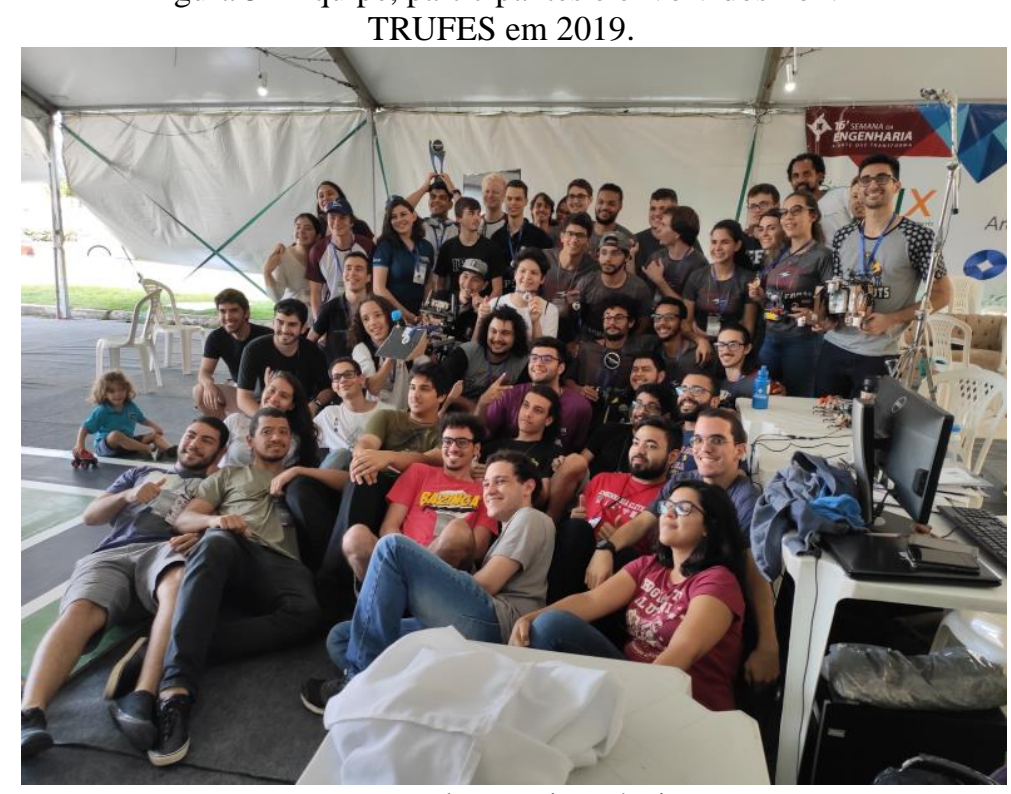

Fonte: de autoria própria.

Em 2020, o TRUFES terá a sua sétima edição e, por consequência das restrições impostas pela pandemia de Covid-19, será realizado inteiramente online pela primeira vez. Para isso, simuladores serão utilizados pela equipe e pelos participantes para avaliar o desempenho da equipe competidora de maneira similar à realizada presencialmente, visando fomentar a robótica capixaba (e até mesmo a nacional, dado que equipes de outros estados também participam do evento) respeitando a prática do distanciamento social durante a pandemia. Essas ações incentivam a participação em competições internacionais, como a Latin American Robotics Competition.

Mantendo-se fiel à sua proposta original de interação, contribuição e apoio à sociedade do Espírito Santo, toda a renda arrecadada com as inscrições do VII TRUFES será doada à Acacci (Associação Capixaba Contra o Câncer Infantil).

Além do TRUFES, uma das contribuições do Programa de Extensão ERUS para a comunidade capixaba é seu envolvimento na OBR (Olimpíada Brasileira de Robótica). Nela a equipe participa como um dos organizadores do evento, recrutando e treinando o núcleo de juízes para a competição. A OBR é um evento que ocorre anualmente e conta com modalidades prática e teórica, propondo diversos desafios a serem solucionados pelos seus participantes, que são alunos do ensino fundamental, médio e técnico integrado. Os primeiros colocados na olimpíada são classificados automaticamente para a etapa nacional e, caso obtenham um desempenho exemplar, podem se qualificar até para a etapa internacional.

Assim como o TRUFES, a OBR conta com a participação de estudantes de diversos grupos sociais e faixas etárias, sendo uma das principais exposições do cenário no contexto capixaba junto ao TRUFES. Outro ponto notável do trabalho realizado nesses eventos é a inclusão de participantes do sexo feminino nas competições e, de acordo com os dados da OBR 2018(MUNDO ROBÓTICA, 2018), elas compoêm 45\% dos competidores, promovendo a igualdade entre os gêneros e a redução dos preconceitos perpetuados historicamente nas áreas associadas à tecnologia (LASTRES, et al., 2004). 
É uma honra para a equipe ter um papel fundamental em um evento que agrega tanto ao cenário da robótica no estado, expondo a área da ciência e engenharia a tantos participantes.

\subsection{Treinamentos Internos e Externos.}

Em adição aos aspectos sociais, há também o foco na formação acadêmica e profissional do membro da equipe. Para tal, diversos minicursos, tutoriais, artigos e documentação de projetos são propostos, atualizando o futuro engenheiro acerca das tecnologias do mercado de trabalho e das ferramentas usadas para desenvolvimento de projetos em equipe, como Git (usado para versionamento de código no processo de desenvolvimento de software), Kicad (usado para o desenvolvimento digital de placas de circuito impresso), dentre outros. Dessa forma, transmitindo o conhecimento dos membros mais antigos aos mais novos e mantendo a continuidade do ciclo de aprendizado dentro da equipe.

Além de proporcionar a propagação dos conhecimentos para as gerações futuras da própria equipe, a ERUS mantém os materiais produzidos completamente online, de forma gratuita, para toda a comunidade. Assim, esses conhecimentos se propagam de forma a atingir a população como um todo, permitindo que as áreas da robótica e tecnologia sejam mais acessíveis. Os cursos da equipe também contribuem para isso, uma vez que realizam diversas edições de forma aberta ao público da Ufes e, até mesmo, a públicos de outras instituições de ensino. Instâncias desses cursos já foram ministradas para instituições de ensino fundamental e médio, como visto na Figura 6, também durante a JACEE e como aula especial do Introcomp organizado pelo PET Engenharia da Computação da Ufes.

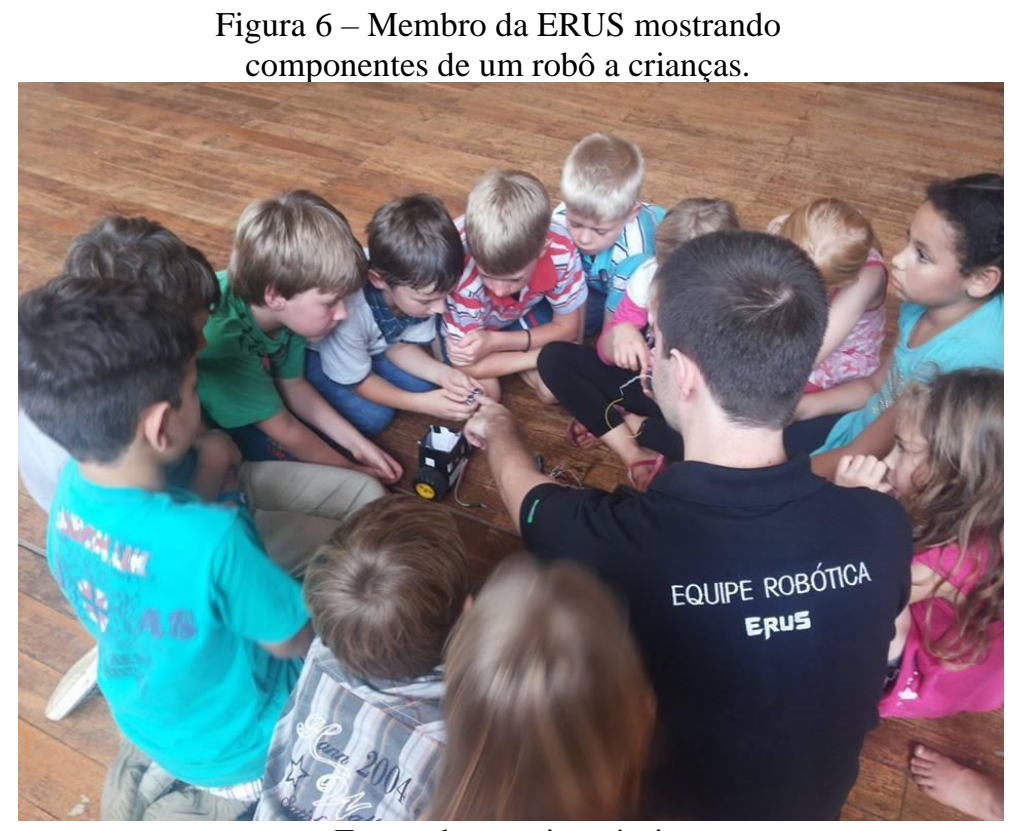

Fonte: de autoria própria.

\section{COMPETIÇÃO: APRENDIZADO NA PRÁTICA E DESENVOLVIMENTO DAS HABILIDADES DO FUTURO ENGENHEIRO (PROBLEM-BASED LEARNING)}

Uma das problemáticas atuais da formação do engenheiro do amanhã é o desenvolvimento da capacidade de analisar e resolver problemas complexos do mundo real. Dentro dos estudos e das técnicas de metodologias pedagógicas para resolver a problemática, vem se tornando comum na literatura o método PBL (Problem-Based Learning). Aplicado 
oficialmente pela primeira vez na área da medicina por meados de 1960 (TIBÉRIO, ATTA, LICHTENSTEIN, 2003), o PBL trata-se de uma metodologia que usa de problemas do mundo real como base para fomentar o interesse e desenvolver a aprendizagem de conceitos (KURT, 2020), procedimentos e atitudes por conta do aluno de forma objetiva (DE CAMARGO RIBEIROA, 2008). Além desse benefício, há outros provenientes do uso dessa técnica, como uma melhor ligação entre os conteúdos aprendidos nos primeiros anos da faculdade e melhor desenvolvimento de habilidades de comunicação para a solução dos problemas (DE CAMARGO RIBEIROA, 2008).

Sabendo dessa importância, a ERUS conta, atualmente, com três times de projetos que são as linhas de frente em participação de competições de robótica nacionais e internacionais. Durante o preparo para as competições de robótica, os alunos são expostos ao método de PBL, onde cada categoria apresenta sua própria problemática e sua gama de assuntos de estudo. São esses times: time de Very Small Size Soccer (VSSS), onde são desenvolvidos robôs autônomos controlados por meio de um computador central que recebe dados de uma câmera como entrada (IEEE, 2020A); time de Line Follower (LF), onde são desenvolvidos robôs que pesam algumas dezenas de gramas e são capazes de se deslocar em cima de uma linha em alta velocidade; e o time do Sumô, onde são desenvolvidos robôs capazes de batalhar autonomamente, sendo uma das categorias mais famosas no cenário da robótica competitiva.

A equipe também já contou com times de outras categorias clássicas, como IEEE-OPEN, desafio que faz os participantes graduandos criarem robôs completamente autônomos para realizarem determinada tarefa que é alterada a cada dois anos (IEEE, 2020B); e IEEE Standard Educational Kit (SEK), que possui os mesmos objetivos da IEEE-OPEN, porém com uso de conjuntos educacionais validados pela competição (IEEE, 2019).

Além dos benefícios ganhos com a abordagem PBL, pelo fato dos alunos serem expostos ao ambiente de desenvolvimento de um projeto com um produto final (o robô), são desenvolvidas outras habilidades, tais como: a gerência de tempo e recursos, uma vez que devem gerir o próprio tempo de trabalho e estudo; a resiliência; o trabalho em equipe; a empatia; a liderança; a autonomia; a proatividade; entre outros. Ademais, a participação em um projeto de extensão favorece a proximidade do aluno a grupos de pesquisa, aos egressos e aos colegas de diferentes áreas da engenharia, podendo resultar em um envolvimento natural na pós-graduação.

O Programa de Extensão ERUS também está bastante articulado com a matriz curricular de vários cursos do Centro Tecnológico da Ufes, realizando apresentações motivacionais em disciplinas como Introdução à Engenharia Elétrica e auxiliando alunos em tarefas práticas como, por exemplo, o desenvolvimento de placas de circuito impresso e manuseio de softwares CAD. Além disso, os alunos que passam pelo Programa dispõem das habilidades ali desenvolvidas como complemento valioso à teoria apresentada nas componentes curriculares de seus cursos.

\section{EM TEMPOS DE COVID-19}

Apesar da situação de pandemia, vivenciada pela população brasileira em decorrência do Covid-19, a ERUS continua trabalhando, junto ao Centro Tecnológico da Ufes, visando o mantenimento de atividades de ensino ao longo da pandemia.

A ERUS se propôs a auxiliar na manutenção de computadores, distribuídos aos discentes que não possuem maneiras de assistir a conteúdos remotos, como aulas virtuais, em virtude de renda insuficiente ou outros problemas externos à universidade, como previsto nas propostas 
de EARTE (Ensino-Aprendizagem Remoto Temporário e Emergencial) externadas pelo grupo de trabalho estruturado pela universidade. Desta forma, a equipe mantém seu pilar de auxílio à comunidade dentro dos limites impostos pela pandemia de maneira segura e efetiva.

\section{NOSSO LEGADO E PRÓXIMOS PASSOS}

A ERUS tem como legado uma quantidade expressiva de ex-membros que fundaram empresas ou que trabalham em áreas afins à robótica em capacidade internacional, além dos que decidiram perseguir a carreira acadêmica. De maneira menos tangível, a ERUS é um agente de mudança à medida que, conforme exposto anteriormente, propicia a vivência com a área da robótica a diversos jovens que, muitas vezes, não teriam contato com esse conteúdo de outras maneiras. Por fim, o desenvolvimento de ferramentas para uso interno usando licenças abertas, como a GPL, viabilizam o uso do trabalho realizado pela equipe por outras pessoas ao redor do mundo.

Para os próximos anos, a ERUS prevê continuidade em sua atual parceria com o Centro Tecnológico da Ufes e os seus diversos cursos de graduação e pós-graduação, expandindo a sua capacidade de desenvolvimento e a relevância dos grupos capixabas de robótica nos cenários nacional e internacional. Além disso, a parceria com outras instituições, como outros grupos de pesquisa e extensão da Ufes ou de outras instituições e empresas externas viabiliza uma ampliação das ações de extensão já realizadas, incrementando o público atingido e refinando a influência que a ERUS tem como agente divulgador da robótica no âmbito estadual e nacional.

\section{Agradecimentos}

A ERUS agradece à direção do Centro Tecnológico da UFES pelo apoio financeiro e administrativo prestado à equipe a todo momento, possibilitando a execução dos projetos descritos neste artigo e o planejamento de projetos futuros por parte da equipe: nada seria possível sem, primeiramente, o espaço físico e infraestrutura proporcionados à equipe para o desenvolvimento dos projetos. Além disso, a ERUS também agradece ao apoio financeiro prestado pela ProEx através de bolsas de extensão e apoio à participação em competições que ocorrem em outros estados.

\section{REFERÊNCIAS}

DE CAMARGO RIBEIROA, Luis Roberto. Aprendizagem baseada em problemas (PBL) na educação em engenharia. Revista de Ensino de Engenharia, v. 27, n. 2, p. 23-32, 2008.

DUCH, Barbara J.; GROH, Susan E.; ALLEN, Deborah E. The power of problem-based learning: a practical" how to" for teaching undergraduate courses in any discipline. Stylus Publishing, LLC., 2001.

IEEE. Regras IEEE Standard Educational Kit 2019, 2019. Disponível em: http://200.145.27.208/cbr/wpcontent/uploads/2020/04/Regras_IEEE_SEK2019_Portugues.pdf. Acesso: 28/07/2020. 


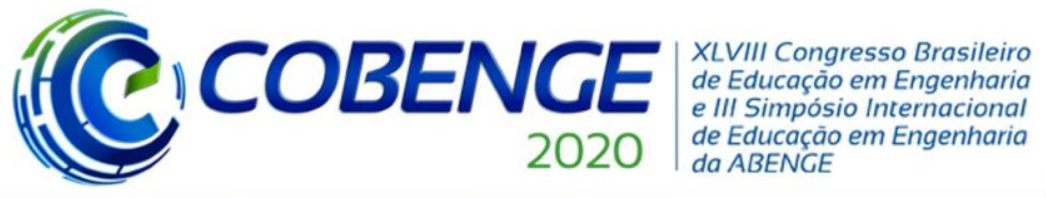

"Os desafios para formar hoje o engenheiro do amanhã"

IEEE. Regras IEEE Very Small Size Soccer (VSSS), 2020. Disponível em: http://200.145.27.208/cbr/wp-content/uploads/2020/07/vssRegras_Portugues.pdf. Acesso: 28/07/2020.

IEEE. RULES OF OPEN Category - 2020/2021, 2020. Disponível em: http://200.145.27.208/cbr/wp-content/uploads/2020/07/OPEN2020_2021_en.pdf. Acesso: 28/07/2020.

KURT, Serhat. Problem-Based Learning (PBL). Educational Technology, 2020. Dispnonível em: https://educationaltechnology.net/problem-based-learning-pbl/. Acesso em: 29/07/2020.

LASTRES, Helena Maria Martins et al. Gênero no sistema de ciência, tecnologia e inovação no Brasil. Gênero, v. 4, p. 73 citation_lastpage= 94, 2004.

MUNDO ROBÓTICA. São Carlos: Universidade Federal de São Carlos, ano 5, n. 13, 2018.

RODRIGUES, Andréia Lilian Lima et al. Contribuições da extensão universitária na sociedade. Caderno de Graduação-Ciências Humanas e Sociais-UNIT-SERGIPE, v. 1, n. 2, p. 141-148, 2013.

TIBÉRIO, Iolanda de FL Calvo; ATTA, José Antonio; LICHTENSTEIN, Arnaldo. O aprendizado baseado em problemas-PBL. Revista de Medicina, v. 82, n. 1-4, p. 78-80, 2003.

\title{
THE ERUS EXTENSION PROGRAM AND ITS CONTRIBUTION IN THE DEVELOPMENT OF THE CAPIXABA ENGINEER
}

\begin{abstract}
This article presents the works of the Ufes Robotics Team and how they contributed for the last eight years to the development of the capixaba engineer, encouraging and allowing students from the Federal University of Espírito Santo to develop their abilities in robotics, computing, mechanics and electronics through the method of Problem-Based Learning.
\end{abstract}

Keywords: Robotics, PBL, Engineering. 